\title{
COHERENT INTERFEROMETRIC IMAGING, TIME GATING AND BEAMFORMING
}

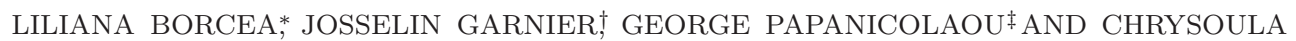 \\ TSOGKA $\S$
}

\begin{abstract}
Coherent interferometric imaging is based on the backpropagation of local space-time cross correlations of array data and was introduced in order to improve images when the medium between the array and the object to be imaged is inhomogeneous and unknown [Borcea et al., Inverse Problems, 21 (2005), 1419]. Although this method has been shown to be effective and is well founded theoretically, the coherent interferometric imaging function is computationally expensive and therefore difficult to use. In this paper we show that this function is equivalent to a windowed beamformer energy function, that is, a quadratic function that involves only time gating and time delaying signals in emission and in reception. In this form the coherent interferometric imaging can be implemented efficiently both in hardware and software, that is, at a computational cost that is comparable to the usual beamforming and migration imaging methods. We also revisit the trade-off between enhanced image stability and loss of resolution in coherent interferometry from the point of view of its equivalence to a windowed beamformer energy imaging function.
\end{abstract}

1. Introduction. When imaging with waves we probe the unknown medium, record the received echoes, the data, and then compute an imaging function. The waves can be acoustic, elastic, or electromagnetic in the microwave, optical or other regimes. They are emitted by one or many sources and they are recorded by sensors, which are transducers such as microphones, seismographs, antennas, etc.

Usually, in sensor array imaging the data are collected first, in the form of the array response matrix, and then they are processed to generate an image at search points (pixels) $\overrightarrow{\boldsymbol{y}}^{S}$ in the image domain $\mathcal{D}$ of interest. The array response matrix is obtained column by column by emitting a pulse from each source location $\overrightarrow{\boldsymbol{x}}_{s}$ at a time and then recording the echoes $P\left(t, \cdot, \overrightarrow{\boldsymbol{x}}_{s}\right)=\left(P\left(t, \overrightarrow{\boldsymbol{x}}_{r}, \overrightarrow{\boldsymbol{x}}_{s}\right)\right)$ at all the receiver locations $\overrightarrow{\boldsymbol{x}}_{r}$. We assume for simplicity that the array has $N$ sensors that are both sources and receivers, and that each source emits the same broadband pulse $f(t)$ (see Figure 1.1). The array response matrix is the $N \times N$ matrix

$$
\mathbb{P}(t)=\left(P\left(t, \overrightarrow{\boldsymbol{x}}_{r}, \overrightarrow{\boldsymbol{x}}_{s}\right)\right)_{r, s=1, \ldots, N}, \quad t \in\left[0, t_{f}\right],
$$

and we call its entries time traces to emphasize that they are functions of time. Here $t$ is a relative time for each column, corresponding to a source illumination, measured after the pulse emission and up to a time $t_{f}$ that is long enough to capture all the echoes from the reflectors to be imaged.

An image can be formed by backpropagating numerically, or migrating, the entries in $\mathbb{P}(t)$ to $\overrightarrow{\boldsymbol{y}}^{S} \in \mathcal{D}$, and then summing over sources and receivers,

$$
\mathcal{I}^{\mathrm{KM}}\left(\overrightarrow{\boldsymbol{y}}^{S}\right)=\sum_{r, s} P\left(\tau\left(\overrightarrow{\boldsymbol{x}}_{r}, \overrightarrow{\boldsymbol{y}}^{S}\right)+\tau\left(\overrightarrow{\boldsymbol{x}}_{s}, \overrightarrow{\boldsymbol{y}}^{S}\right), \overrightarrow{\boldsymbol{x}}_{r}, \overrightarrow{\boldsymbol{x}}_{s}\right) .
$$

This is the well-known Kirchhoff migration approach [2, 3] that backpropagates each trace $P\left(t, \overrightarrow{\boldsymbol{x}}_{r}, \overrightarrow{\boldsymbol{x}}_{s}\right)$ by evaluating it at the travel time $\tau\left(\overrightarrow{\boldsymbol{x}}_{r}, \overrightarrow{\boldsymbol{y}}^{S}\right)+\tau\left(\overrightarrow{\boldsymbol{x}}_{s}, \overrightarrow{\boldsymbol{y}}^{S}\right)$ between the

\footnotetext{
*Computational and Applied Mathematics, MS134, Rice University, 6100 Main Street, Houston, TX 77005-1892 borcea@caam.rice.edu

* Laboratoire de Probabilités et Modèles Aléatoires \& Laboratoire Jacques-Louis Lions, Université Paris VII, 2 Place Jussieu, 75251 Paris Cedex 5, France garnier@math.jussieu.fr

¥Mathematics Department, Stanford University, Stanford, CA 94305 papanicolaou@stanford.edu

$\S$ Applied Mathematics, University of Crete, GR-71409 Heraklion, Greece (tsogka@tem.uoc.gr)
} 
source, the imaging point and the receiver. It works well in known and smooth media but not in heterogeneous, scattering ones, where it produces speckled and unreliable images that are difficult to interpret.

The coherent interferometric (CINT) approach introduced and analyzed in $[6$, $7,10]$, amounts to backpropagating local in time cross correlations of traces at not very distant array locations. It is an effective way to image in scattering media with unknown inhomogeneities (i.e., in clutter), but it is computationally demanding compared to the usual migration methods and therefore of limited use.

An alternative approach to array imaging that may be implemented efficiently in hardware is to form the image by beamforming to each point in the domain $\mathcal{D}$ and forming the image by scanning pixel by pixel. That is, instead of collecting the entire response matrix and then forming the image, the array uses successive multiple illuminations to beamform to each pixel $\overrightarrow{\boldsymbol{y}}^{S} \in \mathcal{D}$, records the echoes and then synchronizes and adds them over the array to form an image at $\overrightarrow{\boldsymbol{y}}^{S}$. The synchronization is done with time delays computed relative to $\overrightarrow{\boldsymbol{y}}^{S}$ and the summation superposes coherently echos from a possible scatterer at $\overrightarrow{\boldsymbol{y}}^{S}$, which is called beamforming in reception. By reciprocity, it is the same as beamforming from the array to $\overrightarrow{\boldsymbol{y}}^{S}$, which is beamforming in emission or illumination. The imaging function at the illuminated pixel is the total field computed over a properly chosen time window and the full image is formed by scanning this way over the image region $\mathcal{D}$.

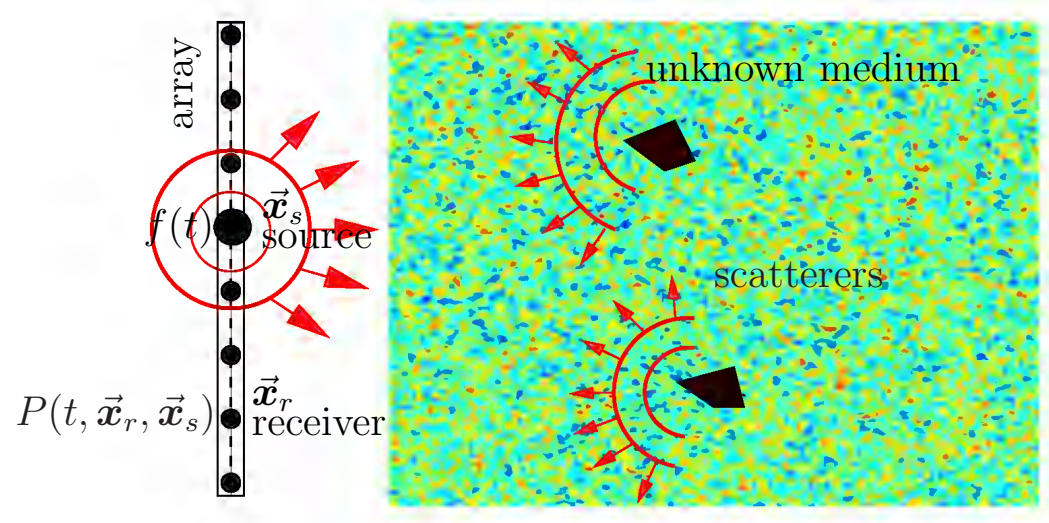

FIG. 1.1. Typical configuration for array imaging.

Beamforming has been used extensively in ultrasonic imaging for non-destructive testing of materials and medical diagnostics [21, 20]. It has also been combined with least squares methods that can improve the images [17, 18]. Beamforming can be readily related to migration methods that allow for some form of optimization of the image, but it does not deal with significant scattering effects in heterogeneous media, which CINT mitigates via an adaptive smoothing. Energy-based beamforming methods, which use quadratic functions of the traces like CINT, have been considered recently [16]. They are still not designed to mitigate scattering in clutter like CINT.

In this paper we show that the CINT function is in fact equivalent to a windowed beamformer energy function, that is, a quadratic function that involves only time gating and time delaying signals in emission and in reception. In this form coherent interferometric imaging can be implemented efficiently both in hardware and software, at a computational cost that is comparable to the usual beamforming and migration imaging methods. We also revisit the trade-off between image stability enhancement 
and loss of resolution in CINT from the point of view of its equivalence to a windowed beamformer energy imaging function.

The paper is organized as follows. We begin in Section 2 with the description of pixel scanning imaging with sub-aperture (windowed) emission and reception beamformers. The equivalence between the mathematical expression of the windowed pixel scanning energy function and the CINT imaging function is presented in Section 3. We also discuss there the relation to two other widely used coherent imaging methods: Kirchhoff migration [2, 3, 13] and matched field imaging [1]. We then explain in Section 4.1 that both the CINT and the windowed beamformer energy functions are, in fact, smoothed Wigner transforms of the data traces, evaluated at the travel times and directions from the sources and receivers to the pixel at $\overrightarrow{\boldsymbol{y}}^{S}$ in the image domain. This formulation allows us to explain in Section 4.2 the role of space-time windowing for achieving robust, statistically stable images in clutter, at the expense of some loss of resolution. We relate briefly in Section 5 the beamformers discussed in this paper to the delay-and-sum ones encountered in the literature. We conclude with a summary in Section 6 .

2. Pixel scanning image formation with windowed energy beamformer. We now describe in detail the scanning imaging process with windowed beamformers in emission and reception. Although the implementation of this process does not require knowledge of the full array response matrix $\mathbb{P}(t)$, we use it here to express mathematically the form of the windowed energy function, which defines the pixel scanning imaging method. We need this model in the next sections to relate the imaging function to those of Kirchhoff migration (KM) and coherent interferometry (CINT).

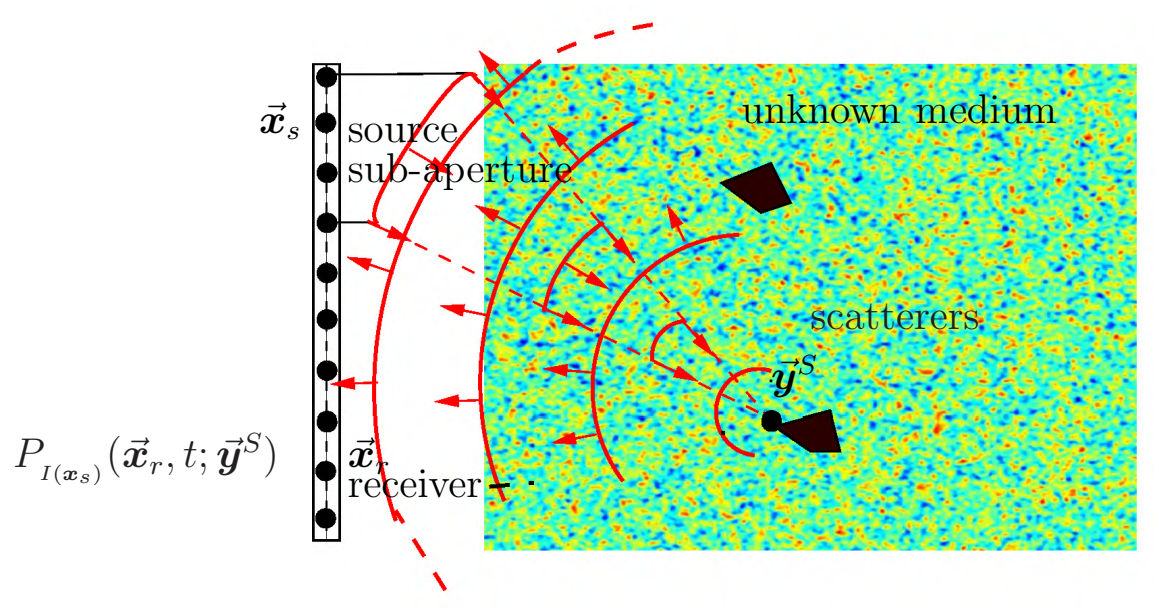

FIG. 2.1. Beamforming configuration for array imaging.

Let $\overrightarrow{\boldsymbol{y}}^{S}$ be an arbitrary pixel in $\mathcal{D}$, at which we form the image. The array beamforms at $\overrightarrow{\boldsymbol{y}}^{S}$ by emitting from its sources delayed pulses. The delays are computed so that all the pulses arrive at $\overrightarrow{\boldsymbol{y}}^{S}$ at the same time. The beamforming can involve all the sources at once, or it can work with sub-apertures (see Figure 2.1). The sources can also be weighted to control the focusing of the beam at $\overrightarrow{\boldsymbol{y}}^{S}$. After beamforming, the array receives the echoes from scattering in the vicinity of $\overrightarrow{\boldsymbol{y}}^{S}$.

In order to have a mathematical model for these echoes, let us suppose for simplicity that the array is linear in the two-dimensional case, or square planar in three 
dimensions. This allows us to introduce a system of coordinates with range axis originating from the array in the orthogonal direction. The sensor locations are $\overrightarrow{\boldsymbol{x}}_{r}=\left(\boldsymbol{x}_{r}, 0\right)$, with $\boldsymbol{x}_{r}$ in the array aperture $\mathcal{A} \subset \mathbb{R}^{n}$, for $r=1, \ldots, N$ and $n=1$ or 2. The aperture is a line segment of length $a$ in two dimensions $(n=1)$ or a square of side $a$ in three dimensions $(n=2)$. We model the sub-apertures and sensor weights used in beamforming with a function $\psi(\xi)$ of dimensionless arguments in $\mathbb{R}^{n}$ and support $|\xi| \leq 1 / 2$ for $n=1$ or $\xi \in[-1 / 2,1 / 2] \times[-1 / 2,1 / 2]$ for $n=2$. The size of the sub-aperture is determined by the length $X$ in the scaled version of $\psi$,

$$
\psi_{X}(\boldsymbol{x})=\psi\left(\frac{\boldsymbol{x}}{X}\right) .
$$

We denote by $P_{I\left(\boldsymbol{x}_{s}\right)}\left(t, \boldsymbol{x}_{r} ; \overrightarrow{\boldsymbol{y}}^{S}\right)$ the echoes received at $\boldsymbol{x}_{r} \in \mathcal{A}$ after beamforming from the sub-aperture centered at $\boldsymbol{x}_{s} \in \mathcal{A}$ on the pixel $\overrightarrow{\boldsymbol{y}}^{S}$. Its expression is

$$
P_{I\left(\boldsymbol{x}_{s}\right)}\left(t, \boldsymbol{x}_{r} ; \overrightarrow{\boldsymbol{y}}^{S}\right)=\sum_{\sigma=1}^{N} \psi_{X}\left(\boldsymbol{x}_{s}-\boldsymbol{x}_{\sigma}\right) P\left(t+\tau\left(\boldsymbol{x}_{\sigma}, \overrightarrow{\boldsymbol{y}}^{S}\right)-\tau\left(\boldsymbol{x}_{s}, \overrightarrow{\boldsymbol{y}}^{S}\right), \overrightarrow{\boldsymbol{x}}_{r}, \overrightarrow{\boldsymbol{x}}_{\sigma}\right),
$$

where we changed slightly the notation of the travel time $\tau\left(\boldsymbol{x}_{s}, \overrightarrow{\boldsymbol{y}}^{S}\right)$ from $\overrightarrow{\boldsymbol{x}}_{s}=\left(\boldsymbol{x}_{s}, 0\right)$ to $\overrightarrow{\boldsymbol{y}}^{S}$, to emphasize its dependence on the cross-range source coordinate $\boldsymbol{x}_{s}$. The relative delays $\tau\left(\boldsymbol{x}_{s}, \overrightarrow{\boldsymbol{y}}^{S}\right)-\tau\left(\boldsymbol{x}_{\sigma}, \overrightarrow{\boldsymbol{y}}^{S}\right)$ are used in (2.2) to synchronize the arrivals at $\overrightarrow{\boldsymbol{y}}^{S}$ of the signals from all the sources in the support of $\psi_{X}$, with center at $\boldsymbol{x}_{s}$. This is the delay-and-sum (DAS) beamforming process $[20,16]$, whose performance depends on the choice of the window function $\psi_{X}$, the frequency band of the probing pulse $f(t)$, and the medium through which the waves propagate.

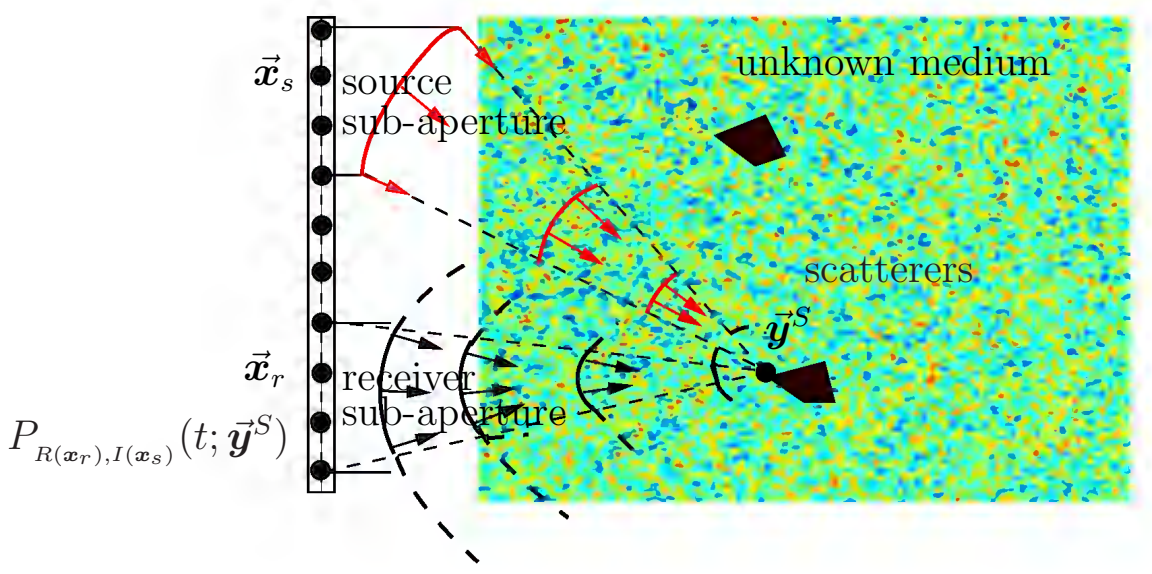

FIG. 2.2. Beamforming configuration for array imaging with sub-apertures.

We assume henceforth that

$$
f(t)=\cos \left(\omega_{o} t\right) f_{B}(t)
$$

with carrier frequency $\omega_{0}$ and baseband pulse $f_{B}(t)$ with Fourier transform $\widehat{f}_{B}(\omega)$ supported at frequencies $|\omega| \leq B / 2$. Then, the frequency band of $f(t)$ is the support $\omega \in\left[\omega_{o}-B / 2, \omega_{o}+B / 2\right] \cup\left[-\omega_{o}-B / 2,-\omega_{o}+B / 2\right]$ of

$$
\widehat{f}(\omega)=\int d t f(t) e^{i \omega t}=\frac{1}{2}\left[\widehat{f}_{B}\left(\omega-\omega_{o}\right)+\widehat{f}_{B}\left(\omega+\omega_{o}\right)\right] .
$$


The larger the bandwidth, the shorter the pulse and the better the range resolution of the beamformer, in smooth and known media, where the arrival of the pulses from the sources can be well synchronized with travel time delays. The cross-range resolution is determined by the carrier wavelength $\lambda_{o}$, the range $L$ of the pixel $\overrightarrow{\boldsymbol{y}}^{S}$ and the window function $\psi_{X}$. When $\psi_{X}=1_{X}$, the indicator function of the subaperture with linear size $X$, the sources are uniformly weighted and the cross-range resolution of the beamformer is of order $\lambda_{o} L / X$, assuming $L \gg X$. Thus, delay and sum beamformers with uniform source weights achieve their best resolution $\lambda_{o} L / a$ by using all the sources at once. The resolution can be improved further by weighting the sources. For example, the results in [10] show that edge illumination, i.e., apodization with a $\Psi_{a}$ peaked near the boundary of the aperture, gives much better cross-range focus at $\overrightarrow{\boldsymbol{y}}^{S}$ than uniform source weighting. Other optimal weight designs for improved focusing and robustness to additive noise are described for example in [21, 17, 18]. However, we have not found in the literature a motivation for using sub-arrays in beamforming. One of the main points of this paper is to show that the aperture thresholding by $X<a$ brings a statistical smoothing that is essential for robustness when imaging in clutter.

The local beamforming in reception consists of synchronizing by travel time delays the received echoes $P_{I\left(\boldsymbol{x}_{s}\right)}\left(t, \boldsymbol{x}_{r} ; \overrightarrow{\boldsymbol{y}}^{S}\right)$ and adding them over the receivers in subapertures centered at $\boldsymbol{x}_{r}$, with uniform or variable weights (see Figure 2.2). We denote the result by $P_{R\left(\boldsymbol{x}_{r}\right), I\left(\boldsymbol{x}_{s}\right)}\left(t ; \overrightarrow{\boldsymbol{y}}^{S}\right)$ and write its mathematical model as follows

$$
P_{R\left(\boldsymbol{x}_{r}\right), I\left(\boldsymbol{x}_{s}\right)}\left(t ; \overrightarrow{\boldsymbol{y}}^{S}\right)=\sum_{\rho=1}^{N} \psi_{X}\left(\boldsymbol{x}_{\rho}-\boldsymbol{x}_{r}\right) P_{I\left(\boldsymbol{x}_{s}\right)}\left(t+\tau\left(\boldsymbol{x}_{\rho}, \overrightarrow{\boldsymbol{y}}^{S}\right)-\tau\left(\boldsymbol{x}_{r}, \overrightarrow{\boldsymbol{y}}^{S}\right), \boldsymbol{x}_{\rho} ; \overrightarrow{\boldsymbol{y}}^{S}\right) .
$$

Note that in general, the weights and sub-aperture may be different in reception than in emission. Here we use the same function $\psi_{X}$ because the sources and receivers coincide in our setup and thus beamforming in reception and emission are mathematically equivalent, by reciprocity. In the case of very large arrays, the windows and weights modeled by $\psi_{X}$ may vary with the center of the sub-apertures. We assume here that the aperture $a$ is small enough so as to use the same window function $\psi_{x}$ for all the emission and reception beamformers.

Naturally, the role of the weights in beamforming in reception is similar to that in emission. For example, the inverse filter developed in [4] achieves the best focusing of the beamformer by emphasizing the edges of the array, just as the edge apodization for emission obtained in [10]. The important point to note is that as in emission, thresholding in reception by a properly chosen $X<a$ introduces a statistical smoothing that is essential for achieving a robust image in clutter. The smaller $X$ is, the more smoothing at the expense of resolution, as discussed in Section 4. Actually, it follows from the analysis of Section 4.1 that the maximum threshold that gives proper smoothing varies with the frequency. Such frequency variation is not considered in the delay-and-sum beamformers because it cannot be implemented in the time domain. Although this frequency dependence may be negligible when the bandwidth $B$ is small compared to the carrier frequency $\omega_{0}$, it may become important when $B \sim \omega_{0}$. Unless we take $X$ as the minimum over the frequency band, which may be too small to achieve an acceptable resolution, robust pixel scanning imaging in clutter may need to be implemented in the frequency domain for broadband pulses, with $X$ variable over the bandwidth.

The last step in the pixel scanning imaging process is to compute the energy of $P_{R\left(\boldsymbol{x}_{r}\right), I\left(\boldsymbol{x}_{s}\right)}\left(t ; \overrightarrow{\boldsymbol{y}}^{S}\right)$ over a properly chosen time window function $\phi_{T}$ and sum over the 
sub-apertures to form the windowed beamforming energy (WBE) imaging function

$$
\mathcal{I}^{\mathrm{WBE}}\left(\overrightarrow{\boldsymbol{y}}^{S} ; T, X\right)=\sum_{s, r} \int \frac{d t}{2 \pi}\left|\phi_{T}\left(t-\tau\left(\boldsymbol{x}_{r}, \overrightarrow{\boldsymbol{y}}^{S}\right)-\tau\left(\boldsymbol{x}_{s}, \overrightarrow{\boldsymbol{y}}^{S}\right)\right) P_{R\left(\boldsymbol{x}_{r}\right), I\left(\boldsymbol{x}_{s}\right)}\left(t ; \overrightarrow{\boldsymbol{y}}^{S}\right)\right|^{2} .
$$

The time window function

$$
\phi_{T}(t)=T^{-1 / 2} \phi\left(\frac{t}{T}\right),
$$

is the scaled version of a function $\phi(u)$ of dimensionless argument that is supported in the interval $|u| \leq 1 / 2$, with normalization so that

$$
\int_{-\infty}^{\infty} \frac{d t}{2 \pi} \phi_{T}^{2}(t)=\int_{-\infty}^{\infty} \frac{d u}{2 \pi} \phi^{2}(u)=\|\phi\|^{2}
$$

The time window is used in (2.6) to evaluate $P_{R\left(\boldsymbol{x}_{r}\right), I\left(\boldsymbol{x}_{s}\right)}\left(t ; \overrightarrow{\boldsymbol{y}}^{S}\right)$ near the travel time $\tau\left(\boldsymbol{x}_{r}, \overrightarrow{\boldsymbol{y}}^{S}\right)+\tau\left(\boldsymbol{x}_{s}, \overrightarrow{\boldsymbol{y}}^{S}\right)$, with precision $T$. This time scale $T$ can account for the pulse delay spread in clutter. In fact, we show in Sections 4.1 and 4 that when $T$ is chosen as the time delay spread, the window function $\phi_{T}$ introduces a statistical smoothing that is essential for robust imaging in clutter.

3. Connections to coherent interferometric imaging. In this section we show how the mathematical expression (2.6) of the windowed beamformer energy function $\mathcal{I}^{\mathrm{WBE}}$ is related to the CINT function introduced in [6] and defined below in Section 3.2.

\subsection{Transformation of $\mathcal{I}^{\mathrm{WBE}}$ into backpropagated local cross-correlations.} We begin by changing variables in the time integral in (2.6)

$$
t \rightsquigarrow t+\tau\left(\boldsymbol{x}_{r}, \overrightarrow{\boldsymbol{y}}^{S}\right)+\tau\left(\boldsymbol{x}_{s}, \overrightarrow{\boldsymbol{y}}^{S}\right),
$$

and using Parseval's identity obtain

$$
\begin{aligned}
\mathcal{I}^{\mathrm{WBE}}\left(\overrightarrow{\boldsymbol{y}}^{S} ; T, X\right) & =\sum_{s, r} \int \frac{d t}{2 \pi}\left|P_{T, X}\left(t, \boldsymbol{x}_{r}, \boldsymbol{x}_{s} ; \overrightarrow{\boldsymbol{y}}^{S}\right)\right|^{2} \\
& =\sum_{s, r} \int d \omega\left|\widehat{P}_{T, X}\left(\omega, \boldsymbol{x}_{r}, \boldsymbol{x}_{s} ; \overrightarrow{\boldsymbol{y}}^{S}\right)\right|^{2} .
\end{aligned}
$$

Here we have defined

$$
P_{T, X}\left(t, \boldsymbol{x}_{r}, \boldsymbol{x}_{s} ; \overrightarrow{\boldsymbol{y}}^{S}\right)=\phi_{T}(t) P_{R\left(\boldsymbol{x}_{r}\right), I\left(\boldsymbol{x}_{s}\right)}\left(t+\tau\left(\boldsymbol{x}_{r}, \overrightarrow{\boldsymbol{y}}^{S}\right)+\tau\left(\boldsymbol{x}_{s}, \overrightarrow{\boldsymbol{y}}^{S}\right) ; \overrightarrow{\boldsymbol{y}}^{S}\right)
$$

and we recall from equations (2.2) and (2.5) that

$$
\begin{gathered}
P_{R\left(\boldsymbol{x}_{r}\right), I\left(\boldsymbol{x}_{s}\right)}\left(t+\tau\left(\boldsymbol{x}_{r}, \overrightarrow{\boldsymbol{y}}^{S}\right)+\right. \\
\left.\tau\left(\boldsymbol{x}_{s}, \overrightarrow{\boldsymbol{y}}^{S}\right) ; \overrightarrow{\boldsymbol{y}}^{S}\right)=\sum_{\rho, \sigma} \psi_{X}\left(\boldsymbol{x}_{\sigma}-\boldsymbol{x}_{s}\right) \psi_{X}\left(\boldsymbol{x}_{\rho}-\boldsymbol{x}_{r}\right) \\
\times P\left(t+\tau\left(\boldsymbol{x}_{\rho}, \overrightarrow{\boldsymbol{y}}^{S}\right)+\tau\left(\boldsymbol{x}_{\sigma}, \overrightarrow{\boldsymbol{y}}^{S}\right), \overrightarrow{\boldsymbol{x}}_{\rho}, \overrightarrow{\boldsymbol{x}}_{\sigma}\right) .
\end{gathered}
$$

In the frequency domain we have the convolution

$$
\begin{aligned}
\widehat{P}_{T, X}\left(\omega, \overrightarrow{\boldsymbol{x}}_{r}, \overrightarrow{\boldsymbol{x}}_{s} ; \overrightarrow{\boldsymbol{y}}^{S}\right)= & \int \frac{d \omega^{\prime}}{2 \pi} \widehat{\phi}_{T}\left(\omega-\omega^{\prime}\right) \sum_{\rho, \sigma} \psi_{X}\left(\boldsymbol{x}_{\sigma}-\boldsymbol{x}_{s}\right) \psi_{X}\left(\boldsymbol{x}_{\rho}-\boldsymbol{x}_{r}\right) \\
& \times \widehat{P}\left(\omega^{\prime}, \overrightarrow{\boldsymbol{x}}_{\rho}, \overrightarrow{\boldsymbol{x}}_{\sigma}\right) \exp \left\{-i \omega^{\prime}\left[\tau\left(\boldsymbol{x}_{\rho}, \overrightarrow{\boldsymbol{y}}^{S}\right)+\tau\left(\boldsymbol{x}_{\sigma}, \overrightarrow{\boldsymbol{y}}^{S}\right)\right]\right\},
\end{aligned}
$$


and the imaging function becomes

$$
\begin{aligned}
\mathcal{I}^{\mathrm{WBE}}\left(\overrightarrow{\boldsymbol{y}}^{S} ; T, X\right)=\sum_{s, r} \sum_{\rho, \rho^{\prime}} \psi_{X}\left(\boldsymbol{x}_{\rho}-\boldsymbol{x}_{r}\right) \psi_{X}\left(\boldsymbol{x}_{\rho^{\prime}}-\boldsymbol{x}_{r}\right) \sum_{\sigma, \sigma^{\prime}} \psi_{X}\left(\boldsymbol{x}_{\sigma}-\boldsymbol{x}_{s}\right) \psi_{X}\left(\boldsymbol{x}_{\sigma^{\prime}}-\boldsymbol{x}_{s}\right) \\
\quad \times \int d \omega \int \frac{d \omega^{\prime}}{2 \pi} \int \frac{d \omega^{\prime \prime}}{2 \pi} \widehat{\phi}_{T}\left(\omega-\omega^{\prime}\right){\widehat{\hat{\phi}_{T}}\left(\omega-\omega^{\prime \prime}\right)}_{P}\left(\omega^{\prime}, \overrightarrow{\boldsymbol{x}}_{\rho}, \overrightarrow{\boldsymbol{x}}_{\sigma}\right) \widehat{\widehat{P}\left(\omega^{\prime \prime}, \overrightarrow{\boldsymbol{x}}_{\rho^{\prime}}, \overrightarrow{\boldsymbol{x}}_{\sigma^{\prime}}\right)} \\
\quad \times \exp \left\{-i \omega^{\prime}\left[\tau\left(\boldsymbol{x}_{\rho}, \overrightarrow{\boldsymbol{y}}^{S}\right)+\tau\left(\boldsymbol{x}_{\sigma}, \overrightarrow{\boldsymbol{y}}^{S}\right)\right]+i \omega^{\prime \prime}\left[\tau\left(\boldsymbol{x}_{\rho^{\prime}}, \overrightarrow{\boldsymbol{y}}^{S}\right)+\tau\left(\boldsymbol{x}_{\sigma^{\prime}}, \overrightarrow{\boldsymbol{y}}^{S}\right)\right]\right\}, \quad(3.5)
\end{aligned}
$$

with the bar denoting complex conjugate.

Now let us use the convolution identity

$$
\int \frac{d \omega}{2 \pi} \widehat{\phi}_{T}\left(\omega-\omega^{\prime}\right) \overline{\widehat{\phi}_{T}\left(\omega-\omega^{\prime \prime}\right)}=\int d t\left|\phi_{T}(t)\right|^{2} e^{i\left(\omega^{\prime \prime}-\omega^{\prime}\right) t}=\widehat{\Phi}\left[T\left(\omega^{\prime \prime}-\omega^{\prime}\right)\right]
$$

that involves the new frequency window function

$$
\widehat{\Phi}(T \omega)=\int d t\left|\phi_{T}(t)\right|^{2} e^{i \omega t}=\int d t|\phi(t)|^{2} e^{i T \omega t},
$$

and introduce also the spatial window function

$$
\begin{aligned}
\Psi\left(\frac{\boldsymbol{x}_{\rho}^{\prime}-\boldsymbol{x}_{\rho}}{X}\right) & =\sum_{r} \psi_{X}\left(\boldsymbol{x}_{\rho}-\boldsymbol{x}_{r}\right) \psi_{X}\left(\boldsymbol{x}_{\rho^{\prime}}-\boldsymbol{x}_{r}\right) \\
& =\sum_{r} \psi\left(\frac{\boldsymbol{x}_{\rho}-\boldsymbol{x}_{r}}{X}\right) \psi\left(\frac{\boldsymbol{x}_{\rho^{\prime}}-\boldsymbol{x}_{\rho}}{X}+\frac{\boldsymbol{x}_{\rho}-\boldsymbol{x}_{r}}{X}\right) .
\end{aligned}
$$

With this notation we obtain the following expression of the windowed beamformer energy function

$$
\begin{aligned}
& \mathcal{I}^{\mathrm{WBE}}\left(\overrightarrow{\boldsymbol{y}}^{S} ; T, X\right)=\frac{1}{2 \pi} \int d \omega \int d \omega^{\prime} \widehat{\Phi}\left[T\left(\omega^{\prime}-\omega\right)\right] \sum_{\rho, \rho^{\prime}} \Psi\left(\frac{\boldsymbol{x}_{\rho^{\prime}}-\boldsymbol{x}_{\rho}}{X}\right) \\
& \times \sum_{\sigma, \sigma^{\prime}} \Psi\left(\frac{\boldsymbol{x}_{\sigma^{\prime}}-\boldsymbol{x}_{\sigma}}{X}\right) \widehat{P}\left(\omega, \overrightarrow{\boldsymbol{x}}_{\rho}, \overrightarrow{\boldsymbol{x}}_{\sigma}\right) \overline{\widehat{P}\left(\omega^{\prime}, \overrightarrow{\boldsymbol{x}}_{\rho^{\prime}}, \overrightarrow{\boldsymbol{x}}_{\sigma^{\prime}}\right)} \\
& \times \exp \left\{-i \omega\left[\tau\left(\boldsymbol{x}_{\rho}, \overrightarrow{\boldsymbol{y}}^{S}\right)+\tau\left(\boldsymbol{x}_{\sigma}, \overrightarrow{\boldsymbol{y}}^{S}\right)\right]+i \omega^{\prime}\left[\tau\left(\boldsymbol{x}_{\rho^{\prime}}, \overrightarrow{\boldsymbol{y}}^{S}\right)+\tau\left(\boldsymbol{x}_{\sigma^{\prime}}, \overrightarrow{\boldsymbol{y}}^{S}\right)\right]\right\} .
\end{aligned}
$$

It follows from this expression that $\mathcal{I}^{\mathrm{WBE}}\left(\overrightarrow{\boldsymbol{y}}^{S} ; T, X\right)$ is mathematically equivalent to the backpropagation to the imaging point $\overrightarrow{\boldsymbol{y}}^{S}$ of the local cross-correlations of the data traces. The backpropagation is done with travel times and the cross-correlations are local because they are computed over receiver and source offsets in the support of the window function $\Psi$ scaled by $X$, and over nearby frequencies satisfying $\left|\omega^{\prime}-\omega\right| \leq$ $1 /(2 T)$.

3.2. Coherent interferometric imaging. The CINT imaging function $\mathcal{I}^{\mathrm{CINT}}$ is similar to $\mathcal{I}^{\mathrm{WBE}}$. Instead of working with the traces themselves, as in the Kirchhoff migration function (1.2), it forms an image by summing over the sources and receivers the local cross-correlations of the traces backpropagated to $\overrightarrow{\boldsymbol{y}}^{S}$ using travel times $[6,7]$

$$
\begin{aligned}
\mathcal{I}^{\mathrm{CINT}}\left(\overrightarrow{\boldsymbol{y}}^{S} ; T^{\mathrm{C}}, X^{\mathrm{C}}\right)=\frac{1}{2 \pi} \int d \omega \int d \omega^{\prime} \widehat{\Phi}^{\mathrm{C}}\left[T^{\mathrm{C}}\left(\omega^{\prime}-\omega\right)\right] \sum_{\rho, \rho^{\prime}} \Psi^{\mathrm{C}}\left(\frac{\boldsymbol{x}_{\rho^{\prime}}-\boldsymbol{x}_{\rho}}{X^{\mathrm{C}}\left(\frac{\omega+\omega^{\prime}}{2}\right)}\right) \\
\quad \times \sum_{\sigma, \sigma^{\prime}} \Psi^{\mathrm{C}}\left(\frac{\boldsymbol{x}_{\sigma^{\prime}}-\boldsymbol{x}_{\sigma}}{X^{\mathrm{C}}\left(\frac{\omega+\omega^{\prime}}{2}\right)}\right) \widehat{P}\left(\omega, \overrightarrow{\boldsymbol{x}}_{\rho}, \overrightarrow{\boldsymbol{x}}_{\sigma}\right) \overline{\widehat{P}\left(\omega^{\prime}, \overrightarrow{\boldsymbol{x}}_{\rho^{\prime}}, \overrightarrow{\boldsymbol{x}}_{\sigma^{\prime}}\right)} \\
\quad \times \exp \left\{-i \omega\left[\tau\left(\boldsymbol{x}_{\rho}, \overrightarrow{\boldsymbol{y}}^{S}\right)+\tau\left(\boldsymbol{x}_{\sigma}, \overrightarrow{\boldsymbol{y}}^{S}\right)\right]+i \omega^{\prime}\left[\tau\left(\boldsymbol{x}_{\rho^{\prime}}, \overrightarrow{\boldsymbol{y}}^{S}\right)+\tau\left(\boldsymbol{x}_{\sigma^{\prime}}, \overrightarrow{\boldsymbol{y}}^{S}\right)\right]\right\} .
\end{aligned}
$$


Here $\widehat{\Phi}^{\mathrm{C}}$ and $\Psi^{\mathrm{C}}$ are the frequency and spatial window functions of dimensionless arguments and finite support. These window functions do not need to be the same as in (3.7) and (3.8), although we show later that it is desirable that they are. The threshold parameters $T^{\mathrm{C}}$ and $X^{\mathrm{C}}$ scale the support of the window functions, and we may let $X^{\mathrm{C}}$ vary with frequency.

By working with local cross-correlations of the data with thresholding $X^{\mathrm{C}}$ and $\Omega^{\mathrm{C}}=1 / T^{\mathrm{C}}$, the sensor separation and frequency offsets, respectively, CINT introduces a statistical smoothing that is designed to stabilize the images with respect to the realizations of the clutter $[8,7]$. The analysis in $[8]$ shows that for $\mathcal{I}^{\mathrm{CINT}}$ to be statistically stable (self-averaging), the thresholding parameters $X^{\mathrm{C}}$ and $\Omega^{\mathrm{C}}$ should not exceed the decoherence length $X_{d}$ and frequency $\Omega_{d}$ of the array data. These parameters characterize how the array data traces decorrelate because of the multiple scattering in clutter. The decoherence frequency $\Omega_{d}$ is the frequency offset $\left|\omega^{\prime}-\omega\right|$ over which $\widehat{P}\left(\omega, \overrightarrow{\boldsymbol{x}}_{r}, \overrightarrow{\boldsymbol{x}}_{s}\right)$ and $\widehat{P}\left(\omega^{\prime}, \overrightarrow{\boldsymbol{x}}_{r}, \overrightarrow{\boldsymbol{x}}_{s}\right)$ become statistically uncorrelated. The decoherence length $X_{d}$ gives the receiver offsets $\left|\boldsymbol{x}_{r^{\prime}}-\boldsymbol{x}_{r}\right|$ over which $\widehat{P}\left(\omega, \overrightarrow{\boldsymbol{x}}_{r}, \overrightarrow{\boldsymbol{x}}_{s}\right)$ and $\widehat{P}\left(\omega, \overrightarrow{\boldsymbol{x}}_{r^{\prime}}, \overrightarrow{\boldsymbol{x}}_{s}\right)$ decorrelate. By reciprocity, the same applies to the source offsets.

In smooth media, the waves do not scatter as they travel from the array to the reflectors that we wish to image and back. There is no loss of coherence of the waves and so there is no need to threshold the sensor and frequency offsets. That is to say, we can take $X^{\mathrm{C}}=a, \Omega^{\mathrm{C}}=B$ and window functions that are equal to one over their support (indicator functions) to obtain from (3.10) and (1.2)

$$
\mathcal{I}^{\mathrm{CINT}}\left(\overrightarrow{\boldsymbol{y}}^{S}\right)=2 \pi\left|\mathcal{I}^{\mathrm{KM}}\left(\overrightarrow{\boldsymbol{y}}^{S}\right)\right|^{2}
$$

The CINT imaging function becomes the square of the Kirchhoff migration one, and there is no gain in working with the cross-correlations of the traces.

Typically, multiple scattering in clutter causes the waves to decorrelate over sensor offsets $X_{d} \ll a$ and small frequency intervals $\Omega_{d}=1 / T_{d} \ll B$, with $T_{d}$ the time delay spread. The decoherence length may be as small as the wavelength, which is why we let $X^{\mathrm{C}}$ be a function of the frequency in (3.10). We discuss this in more detail in Section 4.1, and we describe in Section 4 the trade-off between the resolution of the images and their statistical stability. This trade-off indicates that the smaller the thresholding parameters $X^{\mathrm{C}}$ and $\Omega^{\mathrm{C}}$ are, the more smoothing there is in the images at the expense of loss of resolution. Therefore, it is important to have good estimates of the decoherence length and frequency to obtain relatively sharp and stable images in clutter with $\mathcal{I}^{\text {CINT }}$. The trade-off also motivates an adaptive CINT algorithm, introduced in [7], for estimating the decoherence parameters during the image formation process with CINT. Adaptive CINT iteratively seeks an image with the optimal parameters $X^{\mathrm{C}}$ and $\Omega^{\mathrm{C}}=1 / T^{\mathrm{C}}$, which are also estimates of $X_{d}$ and $\Omega_{d}$, that give the best quality image in terms of resolution and speckle suppression.

3.3. Matched field imaging. Another imaging function that is quadratic in the array data is matched field [1]. The Bartlett matched field imaging function $\mathcal{I}^{\mathrm{MF}}$ can be related to (3.10) in the limit $T^{\mathrm{C}} \rightarrow \infty$ by setting $X^{\mathrm{C}}=a$, with $\Psi^{\mathrm{C}}=1_{a}$ the indicator function of the array,

$$
\mathcal{I}^{\mathrm{MF}}\left(\overrightarrow{\boldsymbol{y}}^{S}\right)=\int \frac{d \omega}{2 \pi}\left|\sum_{\rho, \sigma} \widehat{P}\left(\omega, \overrightarrow{\boldsymbol{x}}_{\rho}, \overrightarrow{\boldsymbol{x}}_{\sigma}\right) e^{-i \omega\left[\tau\left(\boldsymbol{x}_{\rho}, \overrightarrow{\boldsymbol{y}}^{S}\right)+\tau\left(\boldsymbol{x}_{\sigma}, \overrightarrow{\boldsymbol{y}}^{S}\right)\right]}\right|^{2} .
$$

Matched field is designed to mitigate the effect of additive, uncorrelated noise, so it neglects frequency correlations in the data traces. At the same time, it assumes that at 
any frequency $\omega$ in the bandwidth, $\widehat{P}\left(\omega, \overrightarrow{\boldsymbol{x}}_{\rho}, \overrightarrow{\boldsymbol{x}}_{\sigma}\right)$ and $\widehat{P}\left(\omega, \overrightarrow{\boldsymbol{x}}_{\rho^{\prime}}, \overrightarrow{\boldsymbol{x}}_{\sigma^{\prime}}\right)$ remain statistically correlated for all the receivers and sources in the array. In other words, the difference between CINT and matched field is that CINT works with local cross-correlations in time and sensor offsets, whereas matched field takes global cross-correlations, over an infinite time window and the entire array.

Because $\mathcal{I}^{\mathrm{MF}}$ does not exploit the frequency coherence of the array data, it has very poor range resolution in open environments $[11,6]$, unless the array is very large. Range resolution can be recovered only by geometric triangulation using multiple arrays or a single large one, that sees the reflectors to be imaged from very different angles. The imaging function may also lack statistical stability when the decoherence length is much smaller than the array aperture. There are however situations where matched field works well, as in waveguides. The range resolution is recovered in waveguides because of the boundary bouncing modes that give a wide angle view of the scatterer, as if we had a very large array. Matched field, with possible additional data filters [1], works in cluttered waveguides at ranges that are not too long for the wave field to lose its coherence by scattering [5]. This is why it is a popular method in shallow water acoustic [1] imaging.

3.4. Connection between the $\mathcal{I}^{\mathrm{WBE}}$ and $\mathcal{I}^{\mathrm{CINT}}$ imaging functions. We can now state the main result of the paper which relates the pixel scanning windowed beamformer energy function $\mathcal{I}^{\mathrm{WBE}}$ to $\mathcal{I}^{\mathrm{CINT}}$.

TheOREM 3.1. We have

$$
\mathcal{I}^{\mathrm{CINT}}\left(\overrightarrow{\boldsymbol{y}}^{S} ; T^{\mathrm{C}}, X^{\mathrm{C}}\right)=\mathcal{I}^{\mathrm{WBE}}\left(\overrightarrow{\boldsymbol{y}}^{S} ; T, X\right),
$$

if $T^{\mathrm{C}}=T, X^{\mathrm{C}}(\omega)=X$ for all $\omega$ in the bandwidth of the pulse, and if the window functions $\Phi^{\mathrm{C}}$ and $\Psi^{\mathrm{C}}$ in CINT satisfy

$$
\widehat{\Phi}^{\mathrm{C}}(\check{\omega})=\int d u|\phi(u)|^{2} e^{i \check{\omega} u}=\int \frac{d \check{\omega}^{\prime}}{2 \pi} \widehat{\phi}\left(\check{\omega}+\check{\omega}^{\prime}\right) \overline{\widehat{\phi}\left(\check{\omega}^{\prime}\right)},
$$

and

$$
\Psi^{\mathrm{C}}\left(\frac{\boldsymbol{x}_{\rho}^{\prime}-\boldsymbol{x}_{\rho}}{X}\right)=\sum_{r} \psi\left(\frac{\boldsymbol{x}_{\rho}-\boldsymbol{x}_{r}}{X}\right) \psi\left(\frac{\boldsymbol{x}_{\rho^{\prime}}-\boldsymbol{x}_{\rho}}{X}+\frac{\boldsymbol{x}_{\rho}-\boldsymbol{x}_{r}}{X}\right) .
$$

We note that the window functions $\widehat{\Phi}^{\mathrm{C}}$ and $\Psi^{\mathrm{C}}$ are auto-correlation functions and thus have non-negative Fourier transform, by Bochner's theorem. Although we can take, in principle, any window functions in CINT imaging, it is only for autocorrelation functions that the CINT imaging function is non-negative and equal to the energy $\mathcal{I}^{\mathrm{WBE}}$. This observation is important for the adaptive CINT algorithm and for designing optimal filters of the array data for improved focusing of $\mathcal{I}^{\mathrm{WBE}}$ and $\mathcal{I}^{\mathrm{CINT}}$, as pointed out in $[10,9]$. For example, it is shown in $[10,9]$ how to compute optimal subspace projections of the array response matrix in order to minimize the spatial support of the CINT imaging function, and therefore improve the focusing of the image. The small spatial support is obtained by minimizing the $L 1$ norm of $\mathcal{I}^{\text {CINT }}$ normalized by its maximum. By Theorem 3.1 we see that when we restrict the window functions to auto-correlation functions, $\mathcal{I}^{\mathrm{CINT}}$ is non-negative and the $L^{1}$ optimization is in fact one in $L^{2}$.

By relating the pixel scanning beamformer energy function to that of CINT, we can use the statistical stability analysis of $\mathcal{I}^{\mathrm{CINT}}$ [8] to infer the statistical stability of 
$\mathcal{I}^{\mathrm{WBE}}$. Theorem 3.1 says that $\mathcal{I}^{\mathrm{CINT}}$ and $\mathcal{I}^{\mathrm{WBE}}$ are mathematically equivalent only if the sensor offset threshold does not vary over the bandwidth $\left(X^{\mathrm{C}}(\omega)=X\right)$. Then, the results in $[8,7]$, summarized in Section 4.1 , indicate that $\mathcal{I}^{\mathrm{CINT}}$ and therefore $\mathcal{I}^{\mathrm{WBE}}$ is stable with respect to the realizations of the clutter if the thresholding parameters satisfy the bounds

$$
1 / T \leq \Omega_{d}, \quad X \leq X_{d}(\omega),
$$

for all $\omega$ in the pulse bandwidth. Having a statistically stable function $\mathcal{I}^{\mathrm{WBE}}$ means taking a threshold $X$ that is as small as $\min _{\omega} X_{d}(\omega)$, at the expense of blurring the image in cross-range, as explained in Section 4 . Therefore, in broadband cases where $X_{d}(\omega)$ varies significantly, a robust function $\mathcal{I}^{\mathrm{WBE}}$ gives more blurred images in crossrange than adaptive $\mathcal{I}^{\mathrm{CINT}}$, which has built in its design the frequency dependence of the sensor offset threshold.

\section{Stability and resolution analysis.}

4.1. The coherent interferometric imaging function as a smoothed Wigner transform of the array data. In this section we show how the thresholding by $X$ and $T$ introduces a statistical smoothing in the imaging process that is essential for obtaining robust images, relatively insensitive to the realizations of the clutter. Specifically, we show how $\mathcal{I}^{\text {CINT }}$ can be written in terms of the Wigner transform of the array data traces, convolved with the window functions $\Psi$ and $\widehat{\Phi}$. It is because of these convolutions that the smoothing takes place. The analysis is based on the assumption that the sensor separation $h$ in the array is small enough for a continuum aperture approximation to hold, so that we can replace the sums over the array with integrals over the set $\mathcal{A}$,

$$
\sum_{\sigma} \rightsquigarrow \frac{1}{h^{n}} \int_{\mathcal{A}} d \boldsymbol{x}
$$

Let us begin by defining the Wigner transform of the wavefield $P\left(t, \overrightarrow{\boldsymbol{x}}, \overrightarrow{\boldsymbol{x}}^{\prime}\right)$,

$$
\begin{aligned}
\mathcal{W}\left(\bar{\omega}, t ; \overline{\boldsymbol{x}}_{\rho}, \overline{\boldsymbol{x}}_{\sigma} ; \boldsymbol{\kappa}_{\rho}, \boldsymbol{\kappa}_{\sigma}\right)= & \iint_{\mathbb{R}^{n}} d \tilde{\boldsymbol{x}}_{\rho} d \tilde{\boldsymbol{x}}_{\sigma} \int_{-\infty}^{\infty} d \tilde{\omega} \widehat{P}\left(\bar{\omega}+\frac{\tilde{\omega}}{2}, \overline{\boldsymbol{x}}_{\rho}+\frac{\tilde{\boldsymbol{x}}_{\rho}}{2}, \overline{\boldsymbol{x}}_{\sigma}+\frac{\tilde{\boldsymbol{x}}_{\sigma}}{2}\right) \\
& \times \widehat{P}\left(\bar{\omega}-\frac{\tilde{\omega}}{2}, \overline{\boldsymbol{x}}_{\rho}-\frac{\tilde{\boldsymbol{x}}_{\rho}}{2}, \overline{\boldsymbol{x}}_{\sigma}-\frac{\tilde{\boldsymbol{x}}_{\sigma}}{2}\right) e^{-i \tilde{\omega} t+i \bar{\omega} \boldsymbol{\kappa}_{\rho} \cdot \tilde{\boldsymbol{x}}_{\rho}+i \omega \boldsymbol{\kappa}_{\sigma} \cdot \tilde{\boldsymbol{x}}_{\sigma},},
\end{aligned}
$$

where $\boldsymbol{\kappa}_{\rho}$ and $\boldsymbol{\kappa}_{\sigma}$ are slowness vectors in $\mathbb{R}^{n}$. The inverse of this formula, evaluated at mid-points $\overline{\boldsymbol{x}}_{\rho}, \overline{\boldsymbol{x}}_{\sigma} \in \mathcal{A}$ and offsets $\tilde{\boldsymbol{x}}_{\rho}, \tilde{\boldsymbol{x}}_{\sigma}$, reads

$$
\begin{aligned}
\widehat{P}(\bar{\omega}+ & \left.\frac{\tilde{\omega}}{2}, \overline{\boldsymbol{x}}_{\rho}+\frac{\tilde{\boldsymbol{x}}_{\rho}}{2}, \overline{\boldsymbol{x}}_{\sigma}+\frac{\tilde{\boldsymbol{x}}_{\sigma}}{2}\right) \overline{\widehat{P}\left(\bar{\omega}-\frac{\tilde{\omega}}{2}, \overline{\boldsymbol{x}}_{\rho}-\frac{\tilde{\boldsymbol{x}}_{\rho}}{2}, \overline{\boldsymbol{x}}_{\sigma}-\frac{\tilde{\boldsymbol{x}}_{\sigma}}{2}\right)}=\frac{\omega^{2 n}}{(2 \pi)^{1+2 n}} \\
& \times \iint_{\mathbb{R}^{n}} d \boldsymbol{\kappa}_{\rho} d \boldsymbol{\kappa}_{\sigma} \int_{-\infty}^{\infty} d t \mathcal{W}\left(\omega, t ; \overline{\boldsymbol{x}}_{\rho}, \overline{\boldsymbol{x}}_{\sigma} ; \boldsymbol{\kappa}_{\rho}, \boldsymbol{\kappa}_{\sigma}\right) e^{\tilde{\tilde{i} \omega t} t-i \bar{\omega} \boldsymbol{\kappa}_{\rho} \cdot \tilde{\boldsymbol{x}}_{\rho}-i \bar{\omega} \boldsymbol{\kappa}_{\sigma} \cdot \tilde{\boldsymbol{x}}_{\sigma} .}
\end{aligned}
$$

We now use this equation in (3.10) after changing variables

$$
\frac{\boldsymbol{x}_{\rho}+\boldsymbol{x}_{\rho^{\prime}}}{2}=\overline{\boldsymbol{x}}_{\rho}, \quad \boldsymbol{x}_{\rho}-\boldsymbol{x}_{\rho^{\prime}}=\tilde{\boldsymbol{x}}_{\rho} ; \quad \frac{\boldsymbol{x}_{\sigma}+\boldsymbol{x}_{\sigma^{\prime}}}{2}=\overline{\boldsymbol{x}}_{\sigma}, \quad \boldsymbol{x}_{\sigma}-\boldsymbol{x}_{\sigma^{\prime}}=\tilde{\boldsymbol{x}}_{\sigma},
$$

and introducing the central and difference frequencies

$$
\frac{\omega+\omega^{\prime}}{2}=\bar{\omega}, \quad \omega-\omega^{\prime}=\tilde{\omega} .
$$


We then obtain the expression

$$
\begin{aligned}
& \mathcal{I}^{\mathrm{CINT}}\left(\overrightarrow{\boldsymbol{y}}^{S} ; X^{\mathrm{C}}, T^{\mathrm{C}}\right) \approx \frac{h^{-4 n}}{(2 \pi)^{2+2 n}} \iiint d \bar{\omega} d \overline{\boldsymbol{x}}_{\rho} d \overline{\boldsymbol{x}}_{\sigma} \bar{\omega}^{2 n} \\
& \times \iiint d \boldsymbol{\kappa}_{\rho} d \boldsymbol{\kappa}_{\sigma} d t \mathcal{W}\left(\bar{\omega}, t ; \overline{\boldsymbol{x}}_{\rho}, \overline{\boldsymbol{x}}_{\sigma} ; \boldsymbol{\kappa}_{\rho}, \boldsymbol{\kappa}_{\sigma}\right) \\
& \times \int d \tilde{\omega} \widehat{\Phi}^{\mathrm{C}}\left(-T^{\mathrm{C}} \tilde{\omega}\right) \int d \tilde{\boldsymbol{x}}_{\rho} \Psi^{\mathrm{C}}\left(\frac{\tilde{\boldsymbol{x}}_{\rho}}{X^{\mathrm{C}}(\bar{\omega})}\right) \int d \tilde{\boldsymbol{x}}_{\sigma} \Psi^{\mathrm{C}}\left(\frac{\tilde{\boldsymbol{x}}_{\sigma}}{X^{\mathrm{C}}(\bar{\omega})}\right) \\
& \times e^{i \bar{\omega}\left[\tau\left(\overline{\boldsymbol{x}}_{\rho}-\frac{\tilde{\boldsymbol{x}}_{\rho}}{2}, \overrightarrow{\boldsymbol{y}}^{S}\right)-\tau\left(\overline{\boldsymbol{x}}_{\rho}+\frac{\tilde{\boldsymbol{x}}_{\rho}}{2}, \overrightarrow{\boldsymbol{y}}^{S}\right)-\boldsymbol{\kappa}_{\rho} \cdot \tilde{\boldsymbol{x}}_{\rho}+\tau\left(\overline{\boldsymbol{x}}_{\sigma}-\frac{\tilde{\boldsymbol{x}}_{\sigma}}{2}, \overrightarrow{\boldsymbol{y}}^{S}\right)-\tau\left(\overline{\boldsymbol{x}}_{\sigma}-\frac{\tilde{\boldsymbol{x}}_{\sigma}}{2}, \overrightarrow{\boldsymbol{y}}^{S}\right)-\boldsymbol{\kappa}_{\sigma} \cdot \tilde{\boldsymbol{x}}_{\sigma}\right]} \\
& \times e^{i \tilde{\omega}\left[t-\frac{\tau\left(\overline{\boldsymbol{x}}_{\rho}+\frac{\tilde{x}_{\rho}}{2}, \vec{y}^{S}\right)+\tau\left(\overline{\boldsymbol{x}}_{\rho}-\frac{\tilde{x}_{\rho}}{2}, \vec{y}^{S}\right)}{2}-\frac{\tau\left(\overline{\boldsymbol{x}}_{\sigma}+\frac{\tilde{x}_{\sigma}}{2}, \vec{y}^{S}\right)+\tau\left(\overline{\boldsymbol{x}}_{\sigma}-\frac{\tilde{x}_{\sigma}}{2}, \vec{y}^{S}\right)}{2}\right]},
\end{aligned}
$$

which we can simplify under the assumption of small Fresnel numbers

$$
\frac{\omega}{c} \frac{X^{\mathrm{C}}(\omega)^{2}}{\left|\overrightarrow{\boldsymbol{x}}_{\rho}-\overrightarrow{\boldsymbol{y}}^{S}\right|} \sim \frac{\omega}{c} \frac{X^{\mathrm{C}}(\omega)^{2}}{\left|\overrightarrow{\boldsymbol{x}}_{\sigma}-\overrightarrow{\boldsymbol{y}}^{S}\right|} \ll 1
$$

for all $\omega$ in the pulse bandwidth, where $c$ is the constant reference wave speed. This assumption places the calculations in a Fraunhofer diffraction regime where we can linearize the phases in (4.3) and obtain a simpler expression that can be interpreted as a decomposition into plane waves. Indeed, the assumption (4.4) indicates that the sensor separation $X^{\mathrm{C}}$ should by small with respect to $\sqrt{\lambda_{o} L}$, when $L$ is the range from the array to a typical search point where we image. We can then write

$$
\begin{aligned}
& \bar{\omega}\left[\tau\left(\overline{\boldsymbol{x}}_{\rho}-\frac{\tilde{\boldsymbol{x}}_{\rho}}{2}, \overrightarrow{\boldsymbol{y}}^{S}\right)-\tau\left(\overline{\boldsymbol{x}}_{\rho}+\frac{\tilde{\boldsymbol{x}}_{\rho}}{2}, \overrightarrow{\boldsymbol{y}}^{S}\right)\right] \approx-\bar{\omega} \tilde{\boldsymbol{x}}_{\rho} \cdot \nabla_{\overline{\boldsymbol{x}}_{\rho}} \tau\left(\overline{\boldsymbol{x}}_{\rho}, \overrightarrow{\boldsymbol{y}}^{S}\right), \\
& \tilde{\omega}\left[\tau\left(\overline{\boldsymbol{x}}_{\rho}+\frac{\tilde{\boldsymbol{x}}_{\rho}}{2}, \overrightarrow{\boldsymbol{y}}^{S}\right)+\tau\left(\overline{\boldsymbol{x}}_{\rho}-\frac{\tilde{\boldsymbol{x}}_{\rho}}{2}, \overrightarrow{\boldsymbol{y}}^{S}\right)\right] \approx 2 \tilde{\omega} \tau\left(\overline{\boldsymbol{x}}_{\rho}, \overrightarrow{\boldsymbol{y}}^{S}\right),
\end{aligned}
$$

and use the identities

$$
\int d \tilde{\omega} \widehat{\Phi}^{\mathrm{C}}\left(-T^{\mathrm{C}} \tilde{\omega}\right) e^{i \tilde{\omega}\left[t-\tau\left(\overline{\boldsymbol{x}}_{\rho}, \overrightarrow{\boldsymbol{y}}^{S}\right)-\tau\left(\overline{\boldsymbol{x}}_{\sigma}, \overrightarrow{\boldsymbol{y}}^{S}\right)\right]}=\frac{2 \pi}{T^{\mathrm{C}}} \Phi^{\mathrm{C}}\left(\frac{t-\tau\left(\overline{\boldsymbol{x}}_{\rho}, \overrightarrow{\boldsymbol{y}}^{S}\right)-\tau\left(\overline{\boldsymbol{x}}_{\sigma}, \overrightarrow{\boldsymbol{y}}^{S}\right)}{T^{\mathrm{C}}}\right),
$$

and

$$
\begin{aligned}
& \int d \tilde{\boldsymbol{x}}_{\rho} \Psi^{\mathrm{C}}\left(\frac{\tilde{\boldsymbol{x}}_{\rho}}{X(\bar{\omega})}\right) e^{-i \bar{\omega} \tilde{\boldsymbol{x}}_{\rho} \cdot\left[\boldsymbol{\kappa}_{\rho}+\nabla_{\overline{\boldsymbol{x}}_{\rho}} \tau\left(\overline{\boldsymbol{x}}_{\rho}, \overrightarrow{\boldsymbol{y}}^{S}\right)\right]} \\
& =X^{\mathrm{C}}(\bar{\omega})^{n} \widehat{\Psi}^{\mathrm{C}}\left[\bar{\omega} X^{\mathrm{C}}(\bar{\omega})\left(\boldsymbol{\kappa}_{\rho}+\nabla_{\overline{\boldsymbol{x}}_{\rho}} \tau\left(\overline{\boldsymbol{x}}_{\rho}, \overrightarrow{\boldsymbol{y}}^{S}\right)\right)\right]
\end{aligned}
$$

to obtain

$$
\begin{aligned}
\mathcal{I}^{\mathrm{CINT}}\left(\overrightarrow{\boldsymbol{y}}^{S} ; X^{\mathrm{C}}, T^{\mathrm{C}}\right) \approx C \iiint d \bar{\omega} d \overline{\boldsymbol{x}}_{\rho} d \overline{\boldsymbol{x}}_{\sigma}[\bar{\omega} X(\bar{\omega})]^{2 n} \iiint d \boldsymbol{\kappa}_{\rho} d \boldsymbol{\kappa}_{\sigma} d t \\
\times \mathcal{W}\left(\bar{\omega}, t ; \overline{\boldsymbol{x}}_{\rho}, \overline{\boldsymbol{x}}_{\sigma} ; \boldsymbol{\kappa}_{\rho}, \boldsymbol{\kappa}_{\sigma}\right) \Phi^{\mathrm{C}}\left(\frac{t-\tau\left(\overline{\boldsymbol{x}}_{\rho}, \overrightarrow{\boldsymbol{y}}^{S}\right)-\tau\left(\overline{\boldsymbol{x}}_{\sigma}, \overrightarrow{\boldsymbol{y}}^{S}\right)}{T^{\mathrm{C}}}\right) \\
\times \widehat{\Psi}^{\mathrm{C}}\left[\bar{\omega} X^{\mathrm{C}}(\bar{\omega})\left(\boldsymbol{\kappa}_{\rho}+\nabla_{\overline{\boldsymbol{x}}_{\rho}} \tau\left(\overline{\boldsymbol{x}}_{\rho}, \overrightarrow{\boldsymbol{y}}^{S}\right)\right)\right] \\
\times \widehat{\Psi}^{\mathrm{C}}\left[\bar{\omega} X^{\mathrm{C}}(\bar{\omega})\left(\boldsymbol{\kappa}_{\sigma}+\nabla_{\overline{\boldsymbol{x}}_{\sigma}} \tau\left(\overline{\boldsymbol{x}}_{\sigma}, \overrightarrow{\boldsymbol{y}}^{S}\right)\right)\right]
\end{aligned}
$$


with constant $C=h^{-4 n} /\left[(2 \pi)^{1+2 n} T^{\mathrm{C}}\right]$. Here $\widehat{\Psi}^{\mathrm{C}}$ is defined by

$$
\widehat{\Psi}^{\mathrm{C}}(\boldsymbol{k})=\int d \xi e^{-i \boldsymbol{k} \cdot \xi} \Psi^{\mathrm{C}}(\xi)
$$

Equation (4.5) shows that the $\mathcal{I}^{\mathrm{CINT}}$ imaging function is a smoothed version of the Wigner transform of the echoes recorded at the array. It has been used in [8] to prove the statistical stability of CINT imaging in random media. By statistical stability we mean that the relative standard deviation of the imaging function is small, or simply stated, that the images are insensitive to the realization of the random medium. The main point of the statistical stability argument is that although the Wigner transform $\mathcal{W}$ is a randomly fluctuating function, it is weakly self-averaging. That is to say, smooth linear functions of $\mathcal{W}$ are deterministic.

We discuss next the trade-off between the smoothing in (4.5) that makes $\mathcal{I}^{\text {CINT }}$ self-averaging function at the expense of loss of resolution in the image.

4.2. Trade-off between stability enhancement and resolution loss. The amount of smoothing in (4.5) depends on the truncation parameters $T^{\mathrm{C}}$ and $X^{\mathrm{C}}(\omega)$. The smaller $\Omega^{\mathrm{C}}=1 / T^{\mathrm{C}}$ and $X^{\mathrm{C}}$ are, the wider the support of the scaled functions $\Phi^{\mathrm{C}}$ and $\widehat{\Psi}^{\mathrm{C}}$ in (4.5). These window functions are now assumed non-negative to relate $\mathcal{I}^{\mathrm{CINT}}$ to the pixel scanning windowed beamformer energy function $\mathcal{I}^{\mathrm{WBE}}$, as stated in Theorem 3.1.

It is clear from (4.5) that $X^{\mathrm{C}}(\bar{\omega})$ should scale as $1 / \bar{\omega}$ to obtain a frequency independent smoothing over the slowness vectors $\boldsymbol{\kappa}_{\rho}$ and $\boldsymbol{\kappa}_{\sigma}$. This was noted in $[7,8]$, where the decoherence length was defined by

$$
X_{d}(\bar{\omega})=\frac{c}{\bar{\omega} \varkappa_{d}},
$$

with dimensionless $\varkappa_{d}$ quantifying the uncertainty of the direction of arrival of the waves, due to scattering in random media. We therefore let

$$
X^{\mathrm{C}}(\bar{\omega})=\frac{c}{\bar{\omega} \varkappa^{\mathrm{C}}}
$$

for some $\varkappa^{\mathrm{C}}$, and rewrite $(4.5)$ as

$$
\begin{aligned}
& \mathcal{I}^{\mathrm{CINT}}\left(\overrightarrow{\boldsymbol{y}}^{S} ; X^{\mathrm{C}}, T^{\mathrm{C}}\right) \approx C^{\prime} \iiint d \bar{\omega} d \overline{\boldsymbol{x}}_{\rho} d \overline{\boldsymbol{x}}_{\sigma} \iiint d \boldsymbol{\kappa}_{\rho} d \boldsymbol{\kappa}_{\sigma} d t \mathcal{W}\left(\bar{\omega}, t ; \overline{\boldsymbol{x}}_{\rho}, \overline{\boldsymbol{x}}_{\sigma} ; \boldsymbol{\kappa}_{\rho}, \boldsymbol{\kappa}_{\sigma}\right) \\
& \times \Phi^{\mathrm{C}}\left(\frac{t-\tau\left(\overline{\boldsymbol{x}}_{\rho}, \overrightarrow{\boldsymbol{y}}^{S}\right)-\tau\left(\overline{\boldsymbol{x}}_{\sigma}, \overrightarrow{\boldsymbol{y}}^{S}\right)}{T^{\mathrm{C}}}\right) \widehat{\Psi}^{\mathrm{C}}\left[\frac{c\left(\boldsymbol{\kappa}_{\rho}+\nabla_{\overline{\boldsymbol{x}}_{\rho}} \tau\left(\overline{\boldsymbol{x}}_{\rho}, \overrightarrow{\boldsymbol{y}}^{S}\right)\right)}{\varkappa^{\mathrm{C}}}\right] \\
& \times \widehat{\Psi}^{\mathrm{C}}\left[\frac{c\left(\boldsymbol{\kappa}_{\sigma}+\nabla_{\overline{\boldsymbol{x}}_{\sigma}} \tau\left(\overline{\boldsymbol{x}}_{\sigma}, \overrightarrow{\boldsymbol{y}}^{S}\right)\right)}{\varkappa^{\mathrm{C}}}\right],
\end{aligned}
$$

with constant $C^{\prime}=C\left(c / \varkappa^{\mathrm{C}}\right)^{2 n}$. Note that this is no longer the same as $\mathcal{I}^{\mathrm{WBE}}$, because of the frequency dependence of $X^{\mathrm{C}}$. The pixel scanning windowed beamformer is normally implemented in the time domain and cannot handle frequency-dependent sensor offset thresholds.

It follows from (4.6) that the longer $T^{\mathrm{C}}$ and the larger $\varkappa^{\mathrm{C}}$ (i.e., the shorter $X^{\mathrm{C}}$ ) are, the more smoothing there is of the Wigner transform in the imaging function. Smoothing is beneficial for the robustness of the imaging process (statistical stability), 
but it decreases the resolution of the images. To have good range resolution, we would like to use short time windows (small $T^{\mathrm{C}}$ ). Similarly, to focus better in beam forming and get good cross range resolution, we would like to use large sub-array apertures $X^{\mathrm{C}}$, and thus small $\varkappa^{\mathrm{C}}$. This trade-off between smoothing for robustness and resolution shows that it is essential that the truncation parameters $T^{\mathrm{C}}$ and $\varkappa^{\mathrm{C}}$ be chosen appropriately. The optimal choices are given by $T^{\mathrm{C}}=T_{d}$, the power time delay spread, and $\varkappa^{\mathrm{C}}=\varkappa_{d}$, the uncertainty in the direction of arrival $[7,8]$. In practice, these parameters are difficult to estimate directly from the data, so it is better to determine them adaptively, by optimizing over $T^{\mathrm{C}}$ and $\kappa^{\mathrm{C}}$ the quality of the resulting image. This is what is done in adaptive CINT [7].

5. Connections to delay and sum beamforming methods. In this section we discuss connections and stress differences between CINT and well-known beamforming methods used in ultrasound echographic imaging. Our presentation here is, however, placed in a general, interdisciplinary context.

Beamforming may be viewed as a way to spatially filter the array data in order to select signals coming from a particular point $\overrightarrow{\boldsymbol{y}}^{S}$ of the image region. The key step in beamforming is the use of delays in the recorded signals and the selection of the contributions that correspond to the sums of travel times from the source $\overrightarrow{\boldsymbol{x}}_{s}$ to the search point $\overrightarrow{\boldsymbol{y}}^{S}$ and from the search point $\overrightarrow{\boldsymbol{y}}^{S}$ to the receiver $\overrightarrow{\boldsymbol{x}}_{r}$ :

$$
P_{s, r}\left(\overrightarrow{\boldsymbol{y}}^{S}\right)=P\left(\tau\left(\boldsymbol{x}_{r}, \overrightarrow{\boldsymbol{y}}^{S}\right)+\tau\left(\boldsymbol{x}_{s}, \overrightarrow{\boldsymbol{y}}^{S}\right), \overrightarrow{\boldsymbol{x}}_{r}, \overrightarrow{\boldsymbol{x}}_{s}\right) .
$$

Here it is assumed that the emitted probing signal is a very short pulse, so that for each search point only one time sample, at the instant $\tau\left(\boldsymbol{x}_{r}, \overrightarrow{\boldsymbol{y}}^{S}\right)+\tau\left(\boldsymbol{x}_{s}, \overrightarrow{\boldsymbol{y}}^{S}\right)$ is taken from each data sequence. This is a hypothesis that is often made in classical ultrasound beamforming, and although it is often mentioned that it is possible to generalize the model to take more samples into account when the transmitted pulse is more general [23], there is no systematic way to perform this task. It is one of the advantages of CINT that it uses more time samples in an efficient way. Explicitly, it accounts for the time delay spread due to scattering in the medium by evaluating the energy resolved locally over time and directions, i.e., the Wigner transform, in a window of appropriately chosen width $T^{C}$, around the travel time $\tau\left(\boldsymbol{x}_{r}, \overrightarrow{\boldsymbol{y}}^{S}\right)+\tau\left(\boldsymbol{x}_{s}, \overrightarrow{\boldsymbol{y}}^{S}\right)$.

In the delay-and-sum (DAS) beamforming method the delayed samples are simply summed to give the DAS pixel value

$$
\mathcal{I}_{\mathrm{DAS}}\left(\overrightarrow{\boldsymbol{y}}^{S}\right)=\sum_{r, s} P_{s, r}\left(\overrightarrow{\boldsymbol{y}}^{S}\right) .
$$

The DAS method is widely used in ultrasound imaging applications [20] and we note from (1.2) that it is equivalent to Kirchhoff or travel-time migration used in seismology for instance $[2,3,13]$. However it is also noted in the literature that this method has poor resolution and signal-to-noise or signal-to-interference ratio [22]. To compensate for these drawbacks robust beamforming methods have been proposed in which the samples are weighted with pixel-dependent weights $w_{r, s}\left(\overrightarrow{\boldsymbol{y}}^{S}\right)$ prior to summation, which gives the general function

$$
\mathcal{I}_{\mathrm{RB}}\left(\overrightarrow{\boldsymbol{y}}^{S}\right)=\sum_{r, s} w_{s, r}\left(\overrightarrow{\boldsymbol{y}}^{S}\right) P_{s, r}\left(\overrightarrow{\boldsymbol{y}}^{S}\right) .
$$

Two classes of beamformers can be distinguished depending on how the weights are chosen: the data-independent beamformers and the statistically optimum ones. 
The weights in a data-independent beamformer are independent of the array data or data statistics and they are chosen in such a way that the beamforming function presents a specified response for a set of predefined situations. The DAS beamformer is an example of data independent beamforming. Another example is the (frequencydependent) universal inverse filter proposed in [4] which is successful in increasing the resolution when the noise level is low and the array is small and oversampled.

The weights in a statistically optimum beamformer depend on the statistics of the data recorded at the array. These statistics are usually not known so adaptive algorithms must be used to estimate the weights. The goal is to optimize the beamformer response so that the filtered signal contains minimal contributions of waves arriving from points different than the pixel $\overrightarrow{\boldsymbol{y}}^{S}$ at which we form the image, while keeping a unit gain for the signal coming from $\overrightarrow{\boldsymbol{y}}^{S}$. The Capon beamformer [12] is the prototype of statistically optimum beamformers and its generalizations have become increasingly popular $[17,14,18,23,19,22]$. The key steps in the Capon beamformer are the estimation of the covariance matrix of the recorded signals and the regularization of a constrained least squares algorithm for the determination of the optimal weights.

When we compare the classical beamforming methods with the CINT methods, we can make the following observations:

- CINT uses a quadratic function while classical beamforming methods use a linear function of the array data. This function is linear up to the adaptive part which consists in estimating the covariance matrix of the signal. This is an important difference because backpropagation of signal correlations has been shown to be more stable with respect to clutter noise than backpropagation of the signals themselves $[6,15]$.

- The spatial correlation component of CINT roughly corresponds to robust beamforming with certain specific weights that correspond to selecting optimal subapertures near the receivers and sources.

- The time (or frequency) correlation component of CINT is absent in classical beamforming. Indeed this correlation component can only be implemented in the form of a quadratic function. However it plays an important role in selecting the coherent contributions, especially with broadband pulses. It provides a systematic way for selecting the duration of the optimal time window around the expected sum of travel times.

- The adaptive procedure in CINT is reduced to the estimation of the two cut-off parameters $X^{\mathrm{C}}$ and $\Omega^{\mathrm{C}}$ that are common for all pairs of source/receiver. This is in contrast with robust beamforming in which the weights have to be estimated for each pixel. As a result it is possible to implement an adaptive CINT procedure based on the quality of the image itself as shown in [7].

6. Summary. In this paper we have established an equivalence between the coherent interferometric imaging function $\mathcal{I}^{\text {CINT }}$ defined by (3.10) and the pixel scanning beamformer energy function $\mathcal{I}^{\mathrm{WBE}}$ defined by (2.6). The most important consequence of this equivalence is that it allows for an efficient hardware and software implementation of coherent interferometry, using time delays and time gatings, that is, at a computational cost that is comparable to the usual beamforming and migration imaging methods.

Other important consequences of this relationship are that the statistical stability of coherent interferometry proved in [8] is maintained in this new version and that the optimal cut-off parameters used in the implementation can be related to the physical 
parameters of the medium (coherence length and frequency) and can be adaptively estimated as in [7].

The connection between $\mathcal{I}^{\mathrm{CINT}}$ and $\mathcal{I}^{\mathrm{WBE}}$ described in Theorem 3.1 is possible provided that:

- The cut-off functions used in $\mathcal{I}^{\mathrm{CINT}}$ are covariance functions or, equivalently, that their Fourier transforms are nonnegative. Using this type of cut-off functions also ensures that the function $\mathcal{I}^{\mathrm{CINT}}$ is positive valued.

- The cut-off parameter $X^{\mathrm{C}}$ used in $\mathcal{I}^{\mathrm{CINT}}$ is frequency-independent. We know from theoretical considerations that the optimal cut-off parameter $X^{\mathrm{C}}$ depends on the reciprocal of the frequency $[7,8]$. If the cut-off parameter is chosen among the class of frequency-independent parameters, then CINT cannot reach its optimal performance. This shows that coherent interferometry can be optimally implemented with the straightforward time windowing and time gating method presented in this paper, provided that the bandwidth is smaller than the carrier frequency. In the case in which the bandwidth is of the same order as the carrier frequency, the optimal CINT method cannot be implemented as a straightforward time windowing and time gating method and a more flexible, but still practically easy, implementation method is to be found that would allow to achieve the optimal performance of CINT while keeping a low degree of complexity.

Acknowledgment. The work of L. Borcea was partially supported by the Office of Naval Research, grant N00014- 09-1-0290, and by the National Science Foundation, grants DMS-0907746 and DMS-0934594. The work of G. Papanicolaou was partially supported by AFOSR grant FA9550-08-1-0089. The work of C. Tsogka was partially supported by the European Research Council Starting Grant, GA 239959 and the European FP7 Marie Curie International Reintegration Grant MIRG-CT-2007-203438. J. Garnier and G. Papanicolaou thank the Institut des Hautes Études Scientifiques (IHÉS) for its hospitality while this work was completed.

\section{REFERENCES}

[1] A.B. Baggeroer, W.A. Kuperman, and P.N. Mikhalevsky, An overview of matched field methods in ocean acoustics, IEEE Journal of Oceanic Engineering, 18 (1993), pp. 401-424.

[2] B. Biondi, 3D Seismic Imaging, no. 14 in Investigations in Geophysics, Society of Exploration Geophysicists, Tulsa, 2006.

[3] N. Bleistein, J. K. Cohen, and J. W. Stockwell JR., Mathematics of multidimensional seismic imaging, migration, and inversion, Springer, New York, 2001.

[4] L. Borcea, T. Callaghan, J. Garnier, and G. Papanicolaou, A universal filter for enhanced imaging with small arrays, Inverse Problems, 26 (2010), p. 015006(29pp).

[5] L. Borcea, L. Issa, And C. Tsogka, Source localization in random acoustic waveguides, SIAM Multiscale Modeling and Simulations, (in press, 2010).

[6] L. Borcea, G. Papanicolaou, and C. Tsogka, Interferometric array imaging in clutter, Inverse Problems, 21 (2005), pp. 1419-1460.

[7] — Adaptive interferometric imaging in clutter and optimal illumination, Inverse Problems, 22 (2006), pp. 1405-1436.

[8] _ - Asymptotics for the space-time Wigner transform with applications to imaging, Stochastic Differential Equations: Theory and Applications. Volume in Honor of Professor Boris L Rozovskii, PH Baxendale and SV Lototsky editors., Interdisciplinary Mathematical Sciences, 2 (2007).

[9] — Optimal illumination and waveform design for imaging in random media, JASA, 122 (2007), pp. 3507-3518.

[10] _ Optimal waveform design for array imaging, Inverse Problems, 23 (2007), pp. 19732021.

[11] L. Borcea, G. Papanicolaou, C. Tsogka, and J. Berryman, Imaging and time reversal in random media, Inverse Problems, 18 (2002), pp. 1247-1279. 
[12] J. CAPON, High resolution frequency-wavenumber spectrum analysis, Proc. IEEE, 57 (1969), pp. $1408-1418$.

[13] J. F. Claerbout, Fundamentals of geophysical data processing : with applications to petroleum prospecting, CA : Blackwell Scientific Publications, Palo Alto, 1985.

[14] H. Cox, R. M. Zeskind, and M. M. Owen, Robust adaptive beamforming, IEEE Trans. Acoust., Speech, Signal Processing, 35 (1987), pp. 1365-1376.

[15] J.-P. Fouque, J. Garnier, G. Papanicolaou, and K. Solna, Wave propagation and time reversal in randomly layered media, Springer, New York, 2007.

[16] M. Klemm, I. J. Craddock, J. A. Leendertz, and R. Benjamin, Improved delay-and-sum beamforming algorithm for breast cancer detection, International Journal of Antennas and Propagation, Article ID 761402, 9 pages, doi:10.1155/2008/761402 (2008).

[17] T.-J. Shan AND T. KAILATH, Adaptive beamforming for coherent signals and interference, IEEE Transactions on Acoustics, Speech and Signal Processing, ASSP-33 (1985), pp. 527-536.

[18] P. Stoica, Z. Wang, And J. Li, Robust Capon beamforming, IEEE Signal Processing Letters, 10 (2003), pp. 172-175.

[19] J.-F. Synnevag, A. Austeng, And S. Holm, Adaptive beamforming applied to medical ultrasound imaging, IEEE Trans. Ultrasonics, Ferroelectrics, and Frequency Control, 54 (2007), pp. $1606-1613$.

[20] K. E. Thomenius, Evolution of ultrasound beamformers, Proc. IEEE ultrasonics symposium, 2 (1996), pp. $1615-1622$.

[21] B. D. Van Veen And K. Buckley, Beamforming: A versatile approach to spatial filtering, IEEE ASSP Magazine, 5 (1988), pp. 4-24.

[22] F. Vignon And M. R. Burcher, Capon beamforming in medical ultrasound imaging with focused beams, IEEE Trans. Ultrasonics, Ferroelectrics, and Frequency Control, 55 (2008), pp. 619-628.

[23] Z. WANG, J. Li, AND R. Wu, Time-delay- and time-reversal-based robust Capon beamformers for ultrasound imaging, IEEE Trans. Medical Imaging, 24 (2005), pp. 1308-1322. 